Tema: Aciaria Elétrica

\title{
VIABILIDADE DO USO DE PÓ DE ACIARIA PARA PIGMENTAÇÃO DE CONCRETO*
}

\begin{abstract}
Franciéli Mantovani ${ }^{1}$ Marlova Piva Kulakowski ${ }^{2}$

Carlos Alberto Mendes de Moraes ${ }^{3}$

\section{Resumo}

Apesar da primeira preocupação com relação à gestão de resíduos ser a busca pela minimização da geração dos mesmos, há muitos casos de processos produtivos que geram resíduos inevitáveis. A investigação e o desenvolvimento de processos tecnológicos capazes de transformar os resíduos no sentido de reutilizá-los são plenamente justificados e passaram a ser uma realidade. Dentro deste panorama, a reciclagem de resíduos potencialmente perigosos, como o pó de aciaria elétrica PAE, é uma importante questão ambiental. A indústria de pigmentação, na busca de matérias primas alternativas e de menor custo, vem apresentando potencial para absorver resíduos que contenham elevados teores de ferro sob a forma de óxidos. Neste sentido, esta pesquisa objetivou avaliar a viabilidade do uso de PAE para pigmentação da fração argamassa do concreto. Para tanto, foram moldados três traços diferentes, com teores de adição de PAE e de pigmento comercial de $0 \%$ e $5 \%$, mantendo-se uma relação água/aglomerante de 0,4 . Para cada traço moldaramse corpos de prova destinados aos ensaios de resistência à compressão e de medições colorimétricas. A partir dos resultados obtidos, verificou-se que o PAE apresenta potencial para pigmentação de argamassas. Entretanto, verificou-se retardo na pega do material, o que pode vir a prejudicar a desforma.
\end{abstract}

Palavras-chave: Pigmentação; Concreto; Pó de aciaria.

\section{FEASIBILITY OF USING ACIARY POWDER FOR CONCRETE PIGMENTATION}

\section{Abstract}

Although the primary concern about waste management is the search for minimizing its generation, there are many cases of processes that generate inevitable wastes. The research and development of technological processes that transform the waste in order to reuse it are fully justified and became a reality. Within this framework, the recycling of potentially hazardous waste, such as electric arc furnace dust - EAFD, is an important environmental issue. The industry of pigmentation, in the search for alternative raw materials and lower costs, has shown potential to absorb wastes containing high levels of iron in the form of oxides. In this sense, this research aimed to evaluate the feasibility of using EAFD on the pigmentation of the mortar fraction of the concrete. To evaluate it, three different mixes were shaped with levels of addition of EAFD and commercial pigment of $0 \%$ and $5 \%$, maintaining a $\mathrm{w} / \mathrm{b}$ ratio of 0,4 . For each mix were shaped specimens for testing compressive strength and colorimetric measurements. From the results, it was found that the EAFD has potential for pigmentation of mortars. However, it was verified some delay in the handle of the material, which can affect its deformation.

Keywords: Pigmentation; Electric arc furnace dust; Concrete.

1 Arquiteta e Urbanista, mestranda, Universidade do Vale do Rio dos Sinos, São Leopoldo, RS, Brasil.

2 Engenheira Civil, Professora Doutora, Programa de Pós Graduação em Engenharia Civil, Universidade do Vale do Rio dos Sinos, São Leopoldo, RS, Brasil.

3 Engenheiro Metalúrgico, Professor Doutor, Programa de Pós Graduação em Engenharia Civil, Universidade do Vale do Rio dos Sinos, São Leopoldo, RS, Brasil.

* Contribuição técnica ao 450 Seminário de Aciaria - Internacional, 25 a 28 de maio de 2014, Porto Alegre, RS, Brasil. 


\section{INTRODUÇÃO}

O pó de aciaria elétrica (PAE) é um resíduo sólido da indústria siderúrgica, gerado como particulado nas emissões provenientes da fabricação do aço em fornos elétricos a arco. De acordo com a NBR 10004 [1], é classificado como resíduo sólido perigoso - classe I, por isso, a Fundação Estadual de Proteção Ambiental do Estado do Rio Grande do Sul - FEPAM - exige que este resíduo seja estocado em local coberto, protegido das intempéries [2].

Segundo Brehm et al.[2], a caracterização física do PAE é importante para o estudo de alternativas de reciclagem, sendo possível comparar os tamanhos de partículas desse resíduo com o tamanho de partículas do material a ser substituído dentro do processo produtivo.

Conforme Prim et al.[3], a indústria de pigmentação, apresenta potencial para absorver os resíduos da siderurgia, por conterem elevado teor de ferro sob a forma de óxidos e metais. Costa [4] acrescenta que a indústria de pigmentação tem procurado encontrar novas estratégias de concorrência, entre elas a busca de matérias primas alternativas e de menor custo.

A fim de tornar a comparação de cores de determinados objetos mais acessível e precisa, a Comissão Internacional de lluminação (CIE), estabeleceu o espaço cromático Yxy, em 1931 e o espaço colorimétrico L*a*b em 1976, atualmente o mais utilizado. Os sistemas de avaliação numérica da cor baseiam-se na refletância e normalmente representam-na a partir dos parâmetros de tonalidade, saturação e luminosidade [5]. Dado o seu caráter tridimensional, a cor pode ser representada graficamente por um sistema de coordenadas perpendiculares (Figura 1) [6]:

Parâmetro L*: indica o grau de luminosidade, varia entre 0 (preto) e 100 (branco);

Parâmetro $a^{*}$ : indica a cromacidade na direção do verde para o vermelho, onde $a^{*}<0$ maior participação da cor verde, $a^{*}>0$ maior participação da cor vermelha;

Parâmetro $b^{*}$ : indica a cromacidade na direção do azul para o amarelo, onde $b^{*}<0$ maior participação da cor azul; $b^{*}>0$ maior participação da cor amarela [7].

Utilizando-se as coordenadas $L^{*} a^{*} b^{*}$ pode-se comparar a cor de objetos e quantificar suas diferenças, estabelecidas em função da variação destes parâmetros [8].

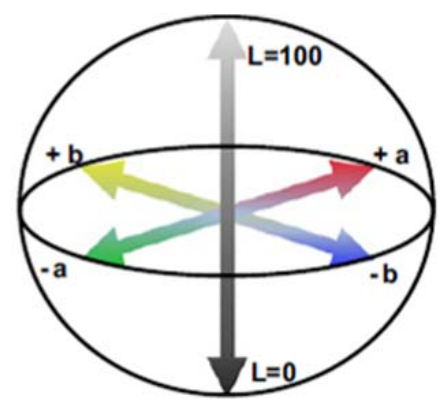

Figura 1. Espaço de cor CIELab [6].

O objetivo deste trabalho é avaliar a viabilidade do uso de pó de aciaria para pigmentação da fração argamassa do concreto.

* Contribuição técnica ao 45 Seminário de Aciaria - Internacional, 25 a 28 de maio de 2014, Porto Alegre, RS, Brasil. 


\section{MATERIAIS E MÉTODOS}

\subsection{Materiais}

O cimento utilizado neste trabalho foi o Portland branco estrutural, classe de resistência de $40 \mathrm{MPa}$. É um cimento de alta resistência inicial, produzido com calcário finamente moído. A Tabela 1 apresenta as características física e mecânica, fornecidas pelo fabricante.

Tabela 1. Caracterização cimento Portland branco estrutural

\begin{tabular}{|c|c|c|c|}
\hline \multicolumn{2}{|c|}{ Caracterização Física } & \multicolumn{2}{c|}{ Resistência à compressão $(\mathrm{MPa})$} \\
\hline Massa Específica $\left(\mathrm{g} / \mathrm{cm}^{3}\right)$ & 3,07 & 1 dia & 25,2 \\
\hline Área Específica $\left(\mathrm{cm}^{2} / \mathrm{g}\right)$ & 4,52 & 3 dias & 42,9 \\
\hline Início de Pega $(\mathrm{h})$ & $03: 30$ & 7 dias & 47,8 \\
\hline Fim de Pega & $04: 25$ & 28 dias & 55,4 \\
\hline
\end{tabular}

Como agregado miúdo utilizou-se areia quartzosa, cuja caracterização física está apresentada na Tabela 2.

Tabela 2. Caracterização física do agregado miúdo

\begin{tabular}{|l|c|r|r|}
\hline \multicolumn{1}{|c|}{ Determinações } & \multicolumn{3}{|c|}{ Resultados obtidos } \\
\hline \multirow{4}{*}{$\begin{array}{c}\text { Composição } \\
\text { granulométrica }\end{array}$} & $\begin{array}{c}\text { Abertura } \\
\text { da peneira }\end{array}$ & \% retida & $\begin{array}{c}\% \\
\text { acumulada }\end{array}$ \\
\cline { 2 - 4 } & 2,4 & 3,5 & 3,5 \\
\cline { 2 - 4 } & 1,2 & 10,0 & 13,5 \\
\cline { 2 - 4 } & 0,6 & 22,5 & 36,0 \\
\cline { 2 - 4 } & 0,3 & 47,0 & 83,0 \\
\cline { 2 - 4 } & 0,15 & 7,5 & 90,5 \\
\cline { 2 - 4 } & $<0,15$ & 9,5 & 100,0 \\
\hline Módulo de finura & \multicolumn{3}{|c}{2,26} \\
\hline Massa específica $\left(\mathrm{kg} / \mathrm{m}^{3}\right)$ & \multicolumn{3}{|c}{2,60} \\
\hline Massa unitária $(\mathrm{kg} / \mathrm{m} 3)$ & \multicolumn{3}{|c}{1,54} \\
\hline
\end{tabular}

A amostra de PAE, que pode ser visualizada na Figura 2, utilizada nesta pesquisa foi caracterizada física e quimicamente.

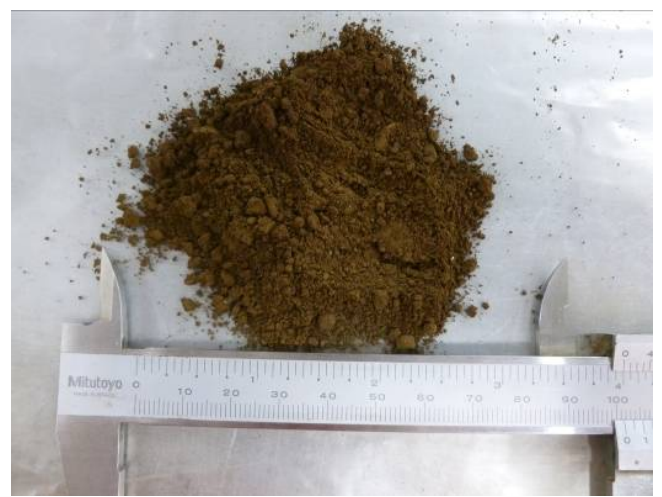

Figura 2. Aspecto visual do PAE em seu estado natural.

\footnotetext{
* Contribuição técnica ao 450 Seminário de Aciaria - Internacional, 25 a 28 de maio de 2014, Porto Alegre, RS, Brasil.
} 
Para que seja possível reaproveitar o PAE na construção civil, como substituto parcial ou como adição ao cimento ou à areia, a análise granulométrica torna-se fundamental. Para isto foi realizada análise via úmida com preparação e gotas de surfactante, fluxo de 10\%, sem ultrassom. Na Figura 3 encontra-se a distribuição do tamanho de partículas onde, $50 \%$ da amostra encontra-se na faixa de tamanho de partícula de até $9,22 \mu \mathrm{m}$.

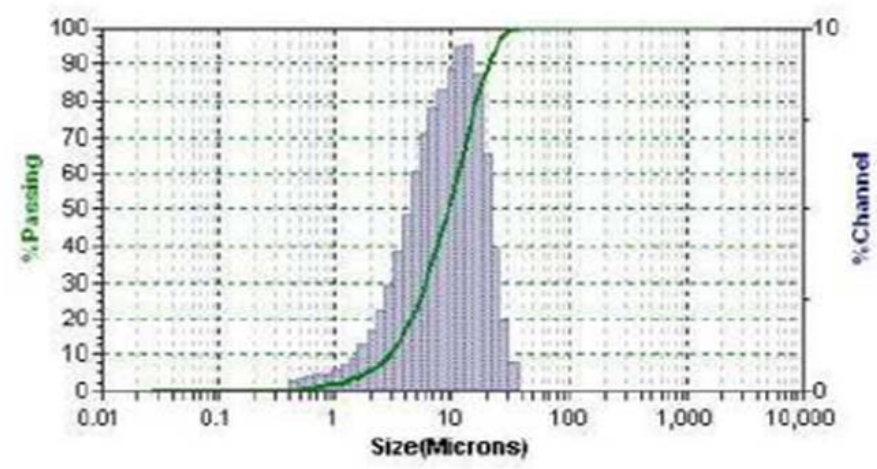

Figura 3. Distribuição granulométrica do PAE por difração a laser.

Apesar de os pigmentos comerciais para uso em concreto possuírem tamanho médio de partícula em torno de $1 \mu \mathrm{m}$ [9], os resultados granulométricos do PAE podem ser considerados favoráveis ao uso.

Para calcular a massa a ser substituída de areia pelo PAE, para que fosse mantido o mesmo volume de materiais no componente, foi necessária a determinação de massa específica. O valor de massa específica encontrado para o PAE foi de 4,09 $\mathrm{g} / \mathrm{cm}^{3}$.

A análise química qualitativa por fluorescência de raios-X do PAE pode ser visualizada na Tabela 3, e apresenta como elemento majoritário, o Ferro.

Tabela 3. Fluorescência de Raios-X do PAE

\begin{tabular}{|c|c|c|}
\hline Elemento majoritário & Elemento minoritário & Elemento traço \\
\hline Ferro (Fe) & Cálcio $(\mathrm{Ca})$ & Chumbo $(\mathrm{Pb})$ \\
\hline & Zinco $(\mathrm{Zn})$ & Silício $(\mathrm{Si})$ \\
\hline & Manganês $(\mathrm{Mn})$ & Cromo $(\mathrm{Cr})$ \\
\hline & & Enxofre (S) \\
\hline & & Potássio (K) \\
\hline & & Cobre (Cu) \\
\hline & & Bromo (Br) \\
\hline & & Níquel $(\mathrm{Ni})$ \\
\hline & & Estrôncio (Sr) \\
\hline
\end{tabular}

A caracterização estrutural da amostra foi realizada através da análise de difração de raios- $x$, apresentada na Figura 3 onde se observa a presença das fases:

1- $\mathrm{MgO}$ (Óxido de Magnésio);

2- $\mathrm{MgFe}_{2} \mathrm{O}_{4}$ (Magnésio-Ferrita);

3- $\mathrm{Fe}_{3} \mathrm{O}_{4}$ (Magnetita).

Observa-se que há uma grande incidência de picos, indicando que sua estrutura é predominantemente cristalina.

\footnotetext{
* Contribuição técnica ao 450 Seminário de Aciaria - Internacional, 25 a 28 de maio de 2014, Porto Alegre, RS, Brasil.
} 

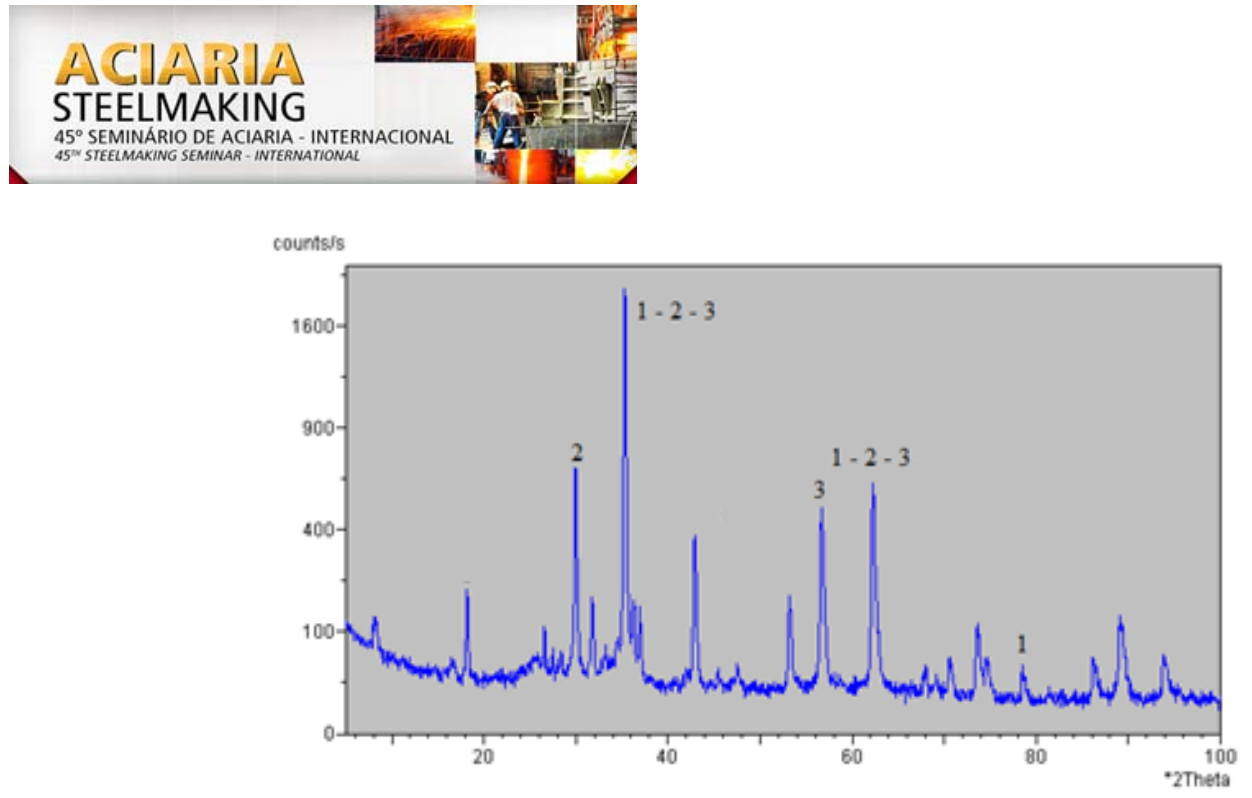

Figura 4. Difratograma de raios-x do PAE.

A perda ao fogo para a amostra de PAE apresentou uma diminuição de $6,57 \%$ da massa. O teor de umidade medido foi de $1,30 \%$.

Com o objetivo de comparar o PAE no que se refere às propriedades de resistência e cor, foi utilizado um pigmento comercial encontrado no mercado, de coloração marrom. É um pigmento inorgânico e, de acordo com o fabricante, a base de óxido de ferro sintético e umidade igual a $0,5 \%$. Encontra-se na forma de pó, com formato de partícula irregular. O ensaio de massa específica apresentou resultado de $4,8 \mathrm{~g} / \mathrm{cm}^{3}$. É insolúvel em água.

\subsection{Metodologia}

Para verificar a viabilidade do uso de PAE em matriz cimentícia, quanto ao comportamento mecânico e colorimétrico, foi desenvolvido um programa experimental descrito na sequência. Os resultados encontrados para o PAE, foram comparados a uma mistura feita somente com cimento e a outra com pigmento comercial encontrado no mercado.

Em função do alto poder de pigmentação dos materiais utilizados, o teor de adição foi fixado em $5 \%$ em relação à massa de cimento, valor recomendado pela bibliografia e pelo fabricante. A quantidade de pigmento ou PAE a ser adicionada à mistura foi compensada pela retirada da mesma quantidade de areia, em volume, para que haja a manutenção do teor de argamassa. Estabeleceu-se relação água/cimento $=0,40$.

No total foram elaboradas três combinações de misturas, sendo que para cada uma foram moldados oito corpos de prova de dimensões $ø 50 \times 100 \mathrm{~mm}$, destinados ao ensaio de resistência à compressão conforme a NBR 5739:2007 [10], rompidos aos 7 e 28 dias; e 4 corpos de prova prismáticos de $80 \times 100 \times 40 \mathrm{~mm}$, destinados ao ensaio colorimétrico.

Os corpos-de-prova permaneceram à temperatura ambiente por 48 horas, com o topo protegido, evitando a perda de umidade. Depois, foram desformados e armazenados em sala climatizada, com temperatura de $\pm 23^{\circ} \mathrm{C}$ e umidade relativa de $60 \%$, onde foram mantidos até a realização do ensaio nas idades especificadas.

\section{RESULTADOS}

Conforme pode ser visualizado na Tabela 4, o índice de consistência do traço de referência obteve resultado de $282 \mathrm{~mm}$. A argamassa que continha pigmento

* Contribuição técnica ao 45 Seminário de Aciaria - Internacional, 25 a 28 de maio de 2014, Porto Alegre, RS, Brasil. 
comercial obteve IC 5,3\% inferior ao da referência, provavelmente justificado pelo formato irregular das partículas, que influenciam mais as propriedades no estado fresco do que no estado endurecido, necessitando de mais pasta de cimento para produzir misturas trabalháveis [11].

Tabela 4. Resultado do índice de consistência

\begin{tabular}{|c|c|}
\hline Traço & IC $(\mathrm{mm})$ \\
\hline Referência & 282 \\
\hline PAE & 284 \\
\hline Pigmento & 267 \\
\hline
\end{tabular}

Ao contrário dos materiais usualmente utilizados em Engenharia, que, quanto menor a granulometria (maior área específica), maior é a quantidade de água necessária para atingir uma mesma trabalhabilidade, o uso do PAE, mesmo com granulometria reduzida, não exigiu maior demanda de água. Brehm et al. [2], avaliaram a morfologia das partículas que compõem o PAE através de microscopia eletrônica de varredura (MEV) por elétrons secundários e observaram que a predominância dos grãos possuem forma esférica e, aparentemente, superfície lisa. Isso pode explicar o alto valor para o IC (284 mm), pois a forma esférica do grão proporciona um efeito lubrificante, por agir como um rolamento.

Entre as características de concretos e argamassas, a resistência mecânica é umas das mais importantes, pois é necessário que o material suporte as cargas a ele submetidas. Além disso, muitas outras propriedades do concreto, como módulo de elasticidade, estanqueidade, resistência às intempéries, estão ligadas a ela, e por isso, podem ser deduzidas a partir dos dados de resistência [11]. Na Figura 5 são apresentados os valores médios da resistência obtida para cada conjunto de quatro corpos de prova, nas idades de 7 e 28 dias.

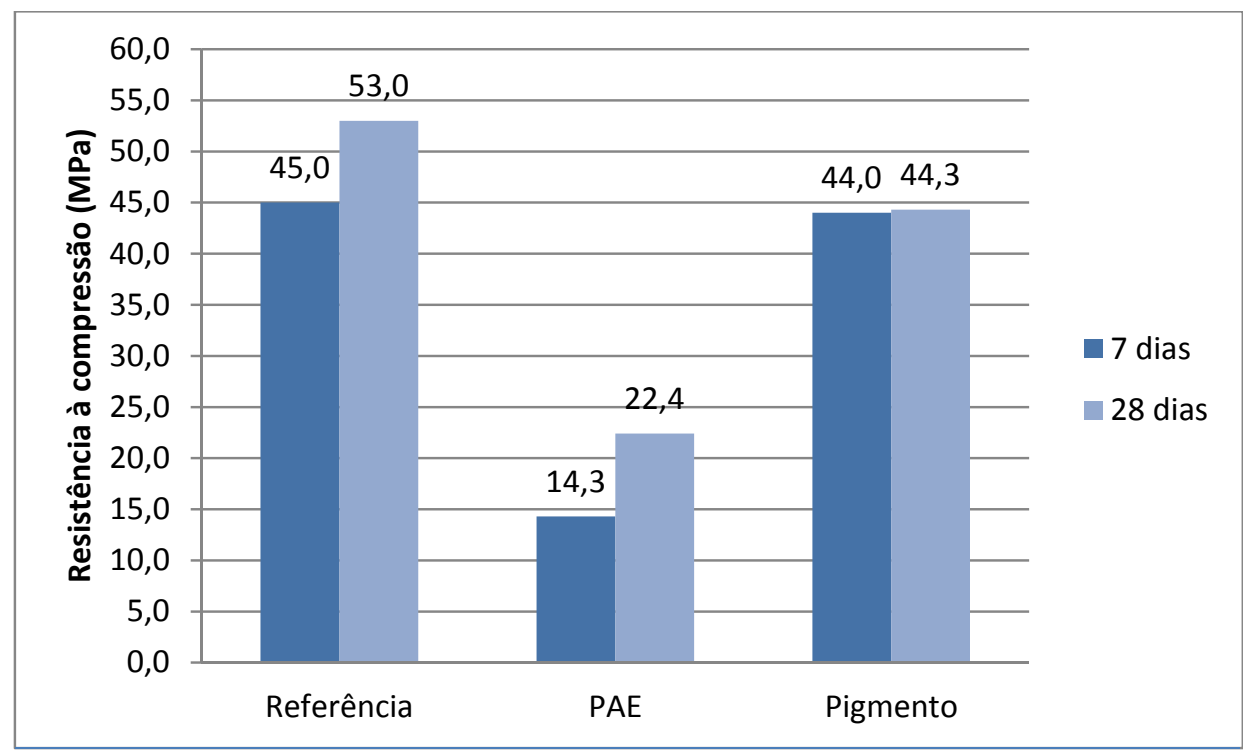

Figura 5. Resultados da média de resistência à compressão aos 7 e 28 dias.

Para uma maior confiabilidade na análise, os dados de resistência obtidos foram submetidos a uma análise de variância (ANOVA) para definição do nível de significância de cada uma das variáveis de controle.

\footnotetext{
* Contribuição técnica ao 45 Seminário de Aciaria - Internacional, 25 a 28 de maio de 2014, Porto Alegre, RS, Brasil.
} 
Nota-se que a resistência da argamassa com adição de PAE decresce $57 \%$ em relação ao traço de referência. Analisando-se estatisticamente, essa diferença é significativa para um nível de confiança de 95\%.

É possível ainda perceber semelhança no comportamento da argamassa com pigmento comercial em relação à referência, aos 7 dias. Aos 28 dias, mesmo com o decréscimo de $16 \%$ em relação ao traço referencia, essa diferença não apresentou ser significativa.

Passuelo [12] afirma que a cor final de uma mistura é obtida através da composição das cores dos materiais utilizados, assim como a dosagem empregada na mistura. Neste trabalho, utilizando-se as coordenadas cromáticas $L^{*} a^{*} b^{*}$, verificou-se apenas a influência do tipo de pigmento e do estado de exposição na cor final.

Dois corpos de prova de cada um dos traços foram submetidos a dois estados de exposição: o testemunho, embalados em plástico filme e tecido de algodão preto, com iluminação, temperatura e umidade controladas; e exposto às internpéries, em ambiente natural.

Com o objetivo de melhor avaliar o comportamento colorimétrico das amostras produzidas e garantir uniformidade na representação da cor, foram realizadas medidas cromáticas utilizando-se o método CIELab, com espectrofotômetro. Esta é uma técnica não destrutiva e permite a obtenção de dados sistematizados. As medições foram realizadas sobre um total de três pontos por corpo de prova na face exposta da amostra, um ao centro e dois nas bordas. As medições foram realizadas aos 2 e 7 dias, com o objetivo de verificar a perda de cor $(\Delta \mathrm{E})$ nas primeiras idades. Os dados colorimétricos obtidos com as leituras espectrofotométricas em cada tipo de mistura estão relacionados na Tabela 5.

Tabela 5. Resultados obtidos na análise de cor, através do espectrofotômetro

\begin{tabular}{|c|c|c|c|c|c|c|c|c|}
\hline \multirow{2}{*}{ Traço } & Tempo & \multicolumn{3}{|c|}{2 dias } & \multicolumn{5}{|c|}{7 dias } \\
\cline { 2 - 9 } & Exposição & $\mathrm{L}^{*}$ & $\mathrm{a}^{*}$ & $\mathrm{~b}^{*}$ & $\mathrm{~L}^{*}$ & $\mathrm{a}^{*}$ & $\mathrm{~b}^{*}$ & $\Delta \mathrm{E}$ \\
\hline \multirow{2}{*}{ Referência } & Testemunho & 82,02 & 0,19 & 7,96 & 84,59 & 0,03 & 6,80 & 2,83 \\
\cline { 2 - 9 } & Natural & 82,45 & 0,13 & 7,72 & 82,72 & 0,29 & 7,37 & 1,21 \\
\hline \multirow{2}{*}{ PAE } & Testemunho & 48,10 & 6,23 & 14,38 & 56,35 & 5,47 & 12,47 & 8,51 \\
\cline { 2 - 9 } & Natural & 48,13 & 6,26 & 14,31 & 57,68 & 5,37 & 12,35 & 9,79 \\
\hline \multirow{2}{*}{ Pigmento } & Testemunho & 37,64 & 12,17 & 10,69 & 38,33 & 11,95 & 10,14 & 1,22 \\
\cline { 2 - 9 } & Natural & 39,79 & 12,60 & 11,03 & 41,53 & 12,09 & 10,09 & 2,08 \\
\hline
\end{tabular}

Observa-se que para o traço de referência, a variação colorimétrica da amostra de testemunho se dá em função da luminosidade. Esta diferença da luminosidade provavelmente é causada por diferenças na cor advindas devido ao processo de cura, tornando mais clara a superfície. As amostras que continham PAE obtiveram os maiores valores de variação, explicado pelo retardo de cura. Aos 2 dias de medição as amostras ainda estavam bastante úmidas. O pigmento comercial, por ser mais estável, apresentou menor variação de cor nos dois estados de exposição. Comparando-se as amostras com algum tipo de pigmento, percebe-se que o traço com PAE produz um crescimento no parâmetro $L^{*}$, indicando que a superfície é mais clara. A incorporação de pigmento comercial provocou uma leve tendência de desvio no parâmetro $a^{*}$ para o vermelho. Já para o parâmetro $b^{*}$, o uso de PAE provocou um desvio para a direção do amarelo.

\footnotetext{
* Contribuição técnica ao 450 Seminário de Aciaria - Internacional, 25 a 28 de maio de 2014, Porto Alegre, RS, Brasil.
} 


\section{CONCLUSÃO}

A partir da análise dos resultados obtidos pode-se concluir que, apesar da queda nos valores de resistência à compressão, causado pelo retardo de pega, a incorporação de PAE apresenta potencial de pigmentação em matriz cimentícia, haja vista a presença de óxidos comumente encontrados nos pigmentos comerciais.

Apesar do tamanho de partícula ser ligeiramente superior ao comumente encontrado nos pigmentos comerciais, o uso de PAE proporcionou uma mistura homogênea, conferindo cor pela dispersão de suas partículas no meio.

Entretanto, os valores de resistência à compressão mostraram-se signficativamente inferiores quando comparados ao traço referência e ao traço com incorporação de pigmento comercial.

\section{REFERÊNCIAS}

1 Associação Brasileira De Normas Técnicas. Resíduos sólidos - classificação: NBR 10004. Rio de Janeiro, 1987.

2 Brehm FA, Machado JGMS, Moraes CAM, Santos CA, Vilela ACF, Cunha JBM. Chemical, physical, structural and morphological characterization of the eletric arc furnace dust. Journal of Hazardous Materials. P. 953-960. 2006

3 Prim SR et al. Synthesis and characterization of hematite pigment obtained from a steel waste industry. Journal of Hazardous Materials, v. 192, n. 3, p. 1307-13, 2011.

4 Costa MGC. Valorização de residuos industriais na formulação de produtos e pigmentos cerâmicos: processamento e desenvolvimento de cor. Tese (Doutorado em Ciência e Engenharia de Materiais). Departamento de Engenharia Cerâmica e do Vidro, Universidade de Aveiro. Portugal. - [S.I.]. 2009.

5 Leite ALSP. Síntese de pigmentos inorgânicos azuis com base em lama de anodização de alumínio. Dissertação (Mestrado). Departamento de Engenharia de Cerâmica e do Vidro. Universidade de Aveiro.Portugal. 2008

6 Korifi R et al. CIELab color space predictive models for colorimetry devices - Analysis of perfume quality. Talanta v. 104, p. 58-66, 2013

7 Vázquez-Nion D. et al. Reliability of color measurements for monitoring pigment content in a biofilm-forming cyanobacterium. International Biodeterioration \& Biodegradation, $p$. 1-7, 2013.

8 Lopez A. et al. Advantages of mortar-based design for coloured self-compacting concrete. Cement and Concrete Composites, v. 31, n. 10, p. 754-761, 2009.

9 Hartmann C, Benini C. Concreto arquitetônico e decorativo. Concreto: Ciência e Tecnologia. 1 ed. 2v. ed São Paulo: IBRACON, 2011. P. 1945-1682.

10 Associação Brasileira de Normas Técnicas. Nbr 5739: Concreto - Ensaios de compressão de corpos-de-prova cilíndricos. Rio de Janeiro, 2007.

11 Mehta PK, Monteiro PJM. Concreto: estrutura, propriedades e materiais. São Paulo: IBRACON, 2008.

12 Passuelo A. Análise de parâmetros influentes na cromacidade e no comportamento mecânico de concretos à base de cimento branco. Dissertação (Mestrado em Engenharia Civil), Escola de Engenharia, Universidade Federal do Rio Grande do Sul Porto Alegre. 2004.

* Contribuição técnica ao 45 Seminário de Aciaria - Internacional, 25 a 28 de maio de 2014, Porto Alegre, RS, Brasil. 